\title{
Domain walls and the conductivity of mesoscopic ferromagnets
}

\author{
Yuli Lyanda-Geller, I. L. Aleiner ${ }^{b}$ and Paul M. Goldbart ${ }^{a}$ \\ ${ }^{a}$ Department of Physics and Materials Research Laboratory, University of Illinois at Urbana-Champaign, Urbana, IL 61801 \\ ${ }^{b}$ NEC Research Institute, Princeton, NJ 08540
}

(13 January 1998)

\begin{abstract}
Quantum interference phenomena in the conductivity of mesoscopic ferromagnets are considered, particularly with regard to the effects of geometric phases acquired by electrons propagating through regions of spatially varying magnetization (due, e.g., to magnetic domain walls). Weak localization and electron-electron interaction quantum corrections to the conductivity and universal conductance fluctuations are discussed. Experiments are proposed for multiply-connected geometries that should reveal conductance oscillations with variations of the profile of the magnetization.
\end{abstract}

PACS numbers: 73.20.Fz, 72.20.Fr, 03.65.Bz

Introduction: In recent experiments, Giordano and Hong [1,2] have investigated the conductivity of ferromagnetic wires containing ferromagnetic domain walls, one aim being to explore the impact of domain walls on mesoscopic quantal phenomena. The purpose of the this Letter is to present a theory of quantum effects in the conductivity of mesoscopic ferromagnets containing domain walls [3], or other magnetic inhomogeneity. Specifically, we address the issue of the electronic resistivity due to ferromagnetic domain walls in disordered samples, and, in particular, study weak-localization and electronelectron interaction (EEI) quantum corrections (QC) to the conductivity, as well as universal conductance fluctuations. We focus on the regime in which the wire width $L_{\perp}$ and the Fermi wave vector $k_{\mathrm{F}}$ satisfy the conditions $W, L_{\perp} \gg l \gg k_{\mathrm{F}}^{-1}, W$ is the thickness of the domain wall. Our approach relies on the fact that domain walls are characterized by an inhomogeneous magnetization (i.e. a self-consistent magnetic field that influences the electron spin), which generates an effective spin-dependent gauge potential that is geometric in character [4]. This gauge potential brings with it a quantal geometric phase [5], in much the same way that an electromagnetic gauge potential brings a quantal Aharonov-Bohm phase. The origin of this geometric phase is the variation of the magnetization encountered by the electron as it propagates through the medium, and its magnitude is determined by the solid angle swept out by the magnetization orientation as the electron propagates (see Fig. 1).

An important, and rather general, issue that we address is the EEI correction to conductivity in the presence of inhomogeneous magnetic fields (either internal or external). As we shall see, for simply-connected geometries the inhomogeneous magnetic field suppresses (the Diffuson channel contribution to) the EEI corrections to conductivity. Moreover, such fields do so at magnitudes lower than those known to a influence the Diffuson channel correction due to homogeneous magnetic fields that couple to the spin. In addition, we address conductance oscillations in inhomogeneous fields in multiply-connected geometries due to EEI. We also analyze the influence of spin-orbit scattering by a random potential (SOI) on Berry-phase effects in inhomogeneous fields, thus extending the consideration of single-particle interference effects in Ref. [7]. To conclude, we discuss the separation of Berry-phase and dynamical-phase effects on conductivity of ferromagnets, and propose feasible experiments in multiply-connected geometries.

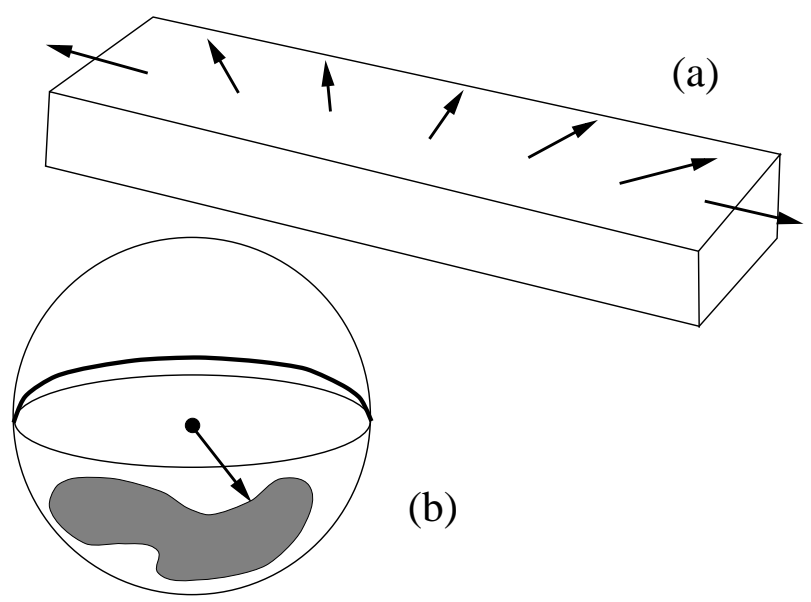

FIG. 1. (a) Schematic depiction of the planar variation of the magnetization in a simple domain wall. (b) Sphere of magnetization orientations, showing the image of the magnetization for this simple domain wall (the near-equatorial thick line), and for a generic magnetization (gray area subtending an arbitrary solid angle at the origin).

It may be worth noting that our approach yields results for the electronic resistivity in the presence of domain wall that are quite different from those recently presented in Ref. [8]. In particular, we find that a single domain wall in which the magnetization direction varies only within a fixed plane leads to neither dephasing nor to changes in the WL corrections to the conductivity. By contrast, two domain walls (or a single domain wall whose magnetization orientation enloses a generic solid angle on the unit sphere) do affect WL conductivity, the essential mechanism being a geometric phase.

The concept of geometric phase can be applied most 
straightforwardly when the relevant adiabatic condition is satisfied. For spins this means that they have sufficient time complete a few precessions before the axis of precession changes significantly. In the present context of the diffusive motion of electrons in ferromagnets, precession is caused by the (spatially varying) spontaneous internal magnetization $\mathbf{M}(\mathbf{r}) /(e g / 2 m c)$ (in which $e, g$ and $m$ are, respectively, the charge, $g$-factor, and free-electron mass) and the adiabatic condition becomes $M \tau \gg\left(\ell / L_{\mathrm{M}}\right)(\ell / \Lambda)$, where $M$ is the characteristic magnitude of $\mathbf{M}(\mathbf{r}), L_{\mathrm{M}}$ is the characteristic length for orientation variations of $\mathbf{M}(\mathbf{r}), \tau$ is the mean free time, and $\Lambda$ is the scale of the diffusive path in question [7]. (For the case of a domain wall, $L_{\mathrm{M}} \sim W$.) This condition is overwhelmingly satisfied when $M \tau \gg 1$, which is the case addressed in Ref. [8], i.e., for $d$ electrons in $\mathrm{Ni}$ or Fe. Moreover, for $s$ electrons, for which $M$ is two orders of magnitude smaller, $M \tau \sim 0.1$, so that the adiabatic condition still holds. Thus, the concept of the Berry phase can be readily applied to the calculation of the resistivity due to the domain wall. Both $s$ - and $d$-electron contributions to the conductivity exhibit Berry phase effects and, as we shall shortly see, these contributions differ in structure, and thus probe distinct aspects of the QC to conductivity - interference corrections and EEI effects. Model and quantum corrections to conductivity: We now consider QC to conductivity in the presense of a domain wall (DW). The effective one-electron Hamiltonian $\mathcal{H}$ of the ferromagnet in the presence of the DW has the form

$$
\mathcal{H}=\sum_{\mathbf{k}, \sigma= \pm} \epsilon_{k} c_{\mathbf{k}, \sigma}^{\dagger} c_{\mathbf{k}, \sigma}+U \sum_{\sigma, \sigma^{\prime}} \int d^{3} x \mathbf{M}(\mathbf{x}) \cdot c_{\mathbf{x}, \sigma}^{\dagger} \sigma_{\sigma \sigma^{\prime}} c_{\mathbf{x}, \sigma^{\prime}}
$$

where $c_{\sigma}$ and $c_{\sigma}^{\dagger}$ are annihilation and creation operator for electrons with $z$-axis spin-projection $\sigma= \pm$, either in position-space $(\mathbf{x})$ or momentum-space $(\mathbf{k}), \epsilon_{k}$ $\left(\equiv \hbar^{2} k^{2} / 2 m^{*}-\epsilon_{\mathrm{F}}\right)$ is the single-electron energy spectrum, relative to the Fermi energy $\epsilon_{\mathrm{F}}, \sigma$ is the Pauli operator, $U$ is the Coulomb interaction (which is self-consistently responsible for the ferromagnetism). To simplify our presentation we adopt units in which $U=1$.

In order to calculate the interference and the EEI corrections to conductivity, and also the universal conductance fluctuations, we need to make use of the relevant Cooperon and Diffuson propagators. By following the standard technique of retaining the subset of the ladder diagrams in disorder-averaged products of (retarded and advanced) Green functions, we readily establish the equations obeyed by the Cooperon $(P=C)$ and Diffuson $(P=D)$ propagators:

$$
\begin{aligned}
{\left[\frac{\partial}{\partial t^{\prime}}+D\right.} & \left\{i \frac{\partial}{\partial \mathbf{x}^{\prime}}+\frac{e}{c} \mathrm{~A}_{P}^{\mathrm{em}}\left(\mathbf{x}^{\prime}\right)\right\}^{2}+\frac{4\left|\mathbf{S}_{P}\right|^{2}}{3 \tau_{\mathrm{so}}} \\
& \left.-i \mathbf{M}_{ \pm}\left(\mathbf{x}^{\prime}\right) \cdot \mathbf{S}_{ \pm}\right] P=\frac{\delta\left(t-t^{\prime}\right) \delta\left(\mathbf{x}-\mathbf{x}^{\prime}\right)}{\pi \nu \tau}
\end{aligned}
$$

where $\mathbf{M}_{ \pm}=\mathbf{M} \pm \tilde{\mathbf{M}}, \mathbf{A}_{C}^{\mathrm{em}}=\mathbf{A}^{\mathrm{em}}+\tilde{\mathbf{A}}^{\mathrm{em}}, \mathbf{A}_{D}^{\mathrm{em}}=$
$\mathbf{A}^{\mathrm{em}}-\tilde{\mathbf{A}}^{\mathrm{em}}, \mathbf{M}$ and $\tilde{\mathbf{M}}, \mathbf{A}^{\mathrm{em}}$ and $\tilde{\mathbf{A}}^{\mathrm{em}}$ are, respectively, the internal magnetization and external electromagnetic vector-potential affecting two particles (1 and $2), \mathbf{S}_{ \pm}=\left(\sigma_{1} \pm \sigma_{2}\right) / 2, \sigma_{1}$ and $\sigma_{2}$ are their Pauli operators, $S_{C}=S_{+}, S_{D}=S_{-}$, and $\tau_{s o}$ is the SOI dephasing time. We note, in passing, that these equations indicate that SOI modifies neither the dynamical Zeeman term nor the effective vector-potential term, terms that were derived in Ref. [7]. Instead, SOI leads only to the usual dephasing of the triplet components of the Cooperon and Diffuson propagators.

We now illustrate how the geometric vector potential $\mathbf{A}^{\mathrm{g}}$ arises due to the spatial variation of $\mathbf{M} / M$. By using the adiabatic criterion we diagonalize Eq. (11) in the spin space for the one-dimensional case and obtain the equation for Diffuson in the form

$$
\left[D\left(i \frac{\partial}{\partial z}-A_{S j}\right)^{2}+i M_{S j}+\frac{4 S}{3 \tau_{\mathrm{so}}}\right] D_{S, j}=\frac{\delta\left(z-z^{\prime}\right)}{\pi \nu \tau}
$$

where $A_{S j}=A^{\mathrm{em}}-\tilde{A}^{\mathrm{em}}+(1-2 S)(1-|j|-j)\left(A^{\mathrm{g}}-(-1)^{j} \tilde{A}^{\mathrm{g}}\right)$, $M_{S j}=M+(-1)^{j} \tilde{M}, S$ is the net spin, and $j$ is its projection [9]. For $\mathbf{M}=M(\sin \beta \cos \gamma, \sin \beta \sin \gamma, \cos \gamma), A^{\mathrm{g}}$ is given by $A^{\mathrm{g}}=(\cos \beta(z)-1) \partial_{z} \gamma / 2$. In the case $\mathbf{A}^{\mathrm{g}}=\tilde{\mathbf{A}}^{\mathrm{g}}$ $(\mathbf{M}=\tilde{\mathbf{M}})$, which is relevant to the interference and EEI correction to the conductivity, the variation of $\mathbf{M}$ results in a geometric vector-potential that enters the expression for the triplet component $(S=1)$ of the Cooperon (Diffusion) propagator for $j= \pm 1$. In the Diffuson propagator, the $j= \pm 1$ components are also affected by the dynamical phase arising from the Zeeman effect of the magnetization [ $M$-term in Eq. 2], which exists even in the case of uniform $\mathrm{M} / M$. Furthermore, all $S=1$ components of the Diffuson propagator are affected by isotropic SOI dephasing, whereas the singlet component $(S=0)$ is not influenced by any of the spin interactions. In the Cooperon propagator, the dynamical Zeeman phase does not affect the $j= \pm 1$ Cooperon components $C_{1, \pm}(M=\tilde{M})$, but these components are influenced by the $\mathbf{A}^{\mathrm{g}}$ and SOI. Instead, the Zeeman dynamical phase mixes $C_{00}$ with the $C_{1,0}$ component.

We now turn to the WL correction to the conductivity, which is determined by the Cooperon propagator. In the absence of SOI, the WL correction is not affected by the Zeeman dynamical phase, owing to the cancellation between the contributions from the $S=0$ and the $(S, j)=(1,0)$ components. In the presence of SOI, the Zeeman dynamical phase leads to a positive contribution to the magnetoresistance (MR), owing to the noncancellation of these components. We note, however, that in the presence of a domain wall in which $|\mathbf{M}|$ is uniform, the dynamical phase does not contribute to the resistancechange because the magnitude of this contribution is the same in the presence and absence of the domain wall. By contrast, the geometric phase, if any there be, owes 
its existence entirely to the presence of the domain wall, and the corresponding MR is determined by the geometric vector-potential.

Let us consider the implications of the geometric vector-potential in various experimental settings in (1) simply- and (2) multiply-connected samples. (1-a) Consider a simply-connected mesoscopic wire, in which the magnetization varies along the wire but not transverse to it. Such magnetizations are is not uncommon and, indeed, correspond to the one considered in Ref. [8]. WL trajectories are closed (in position space) and, therefore, for such magnetizations, enclose no geometric flux. Thus, such spatial variation of the magnetization generates no contribution to MR. The result of the present work differs from that found in Ref. 8]. [As $\mathbf{M}$ varies on lengthscales much larger than $\ell$ in the setting of interactions with domain walls, disorder-averaging was performed incorrectly in Ref. [8].] (1-b) Suppose, instead, that there is some transverse variation of the magnetization. Then the image of $\mathbf{M}$ covers some area on the unit sphere of M-orientations. In this case, WL trajectories can include geometric flux, and therefore there is a contribution to MR. Note that in two-dimensional ferromagnets, topological excitations of magnetic order, such as skyrmions (i.e. two-dimensional textures), may lead to the modulation of the conductivity owing to the possibility of electron trajectories enclosing differing amounts of geometric flux. (2) Now consider a multiply-connected mesoscopic wire, in which $\mathbf{M}$ varies along the wire but not transverse to it. In this case, multiply-connected WL trajectories enclose multiples of the effective geometric flux threading the hole, and therefore there will be a contribution to MR that oscillates with the flux. For example, such a flux-producing setting would arise in a ring containing two domain walls, arranged so that the variation M-orientation around the ring encloses a nontrivial solid angle (and, ideally, is tunable, e.g., via a magnetic field). In both cases, 1 and 2 , if $\mathbf{M} / M$ varies transverse to the wire then there will be a relative dephasing of formerly coherent WL trajectories, which, in the first case leads to a negative MR, and in the second case results in the decay of the oscillations with geometric flux.

Electron-electron interaction effects: We now consider the EEI contribution to the QC to the conductivity in the presence of inhomogeneous magnetization orientations. QC to various kinetic and thermodynamic quantities, due to EEI in disordered conductors, have their origin in the enhancement of interactions between electrons. The main contribution to this enhancement is due to electron diffusion, which results in an increase of the effective interaction time, and therefore an increase of the effective interaction strength, that is important for electrons having close momenta and energies. This enhancement is described by QC in the Diffuson channel. Various factors that affect electronic coherence (such as inhomogeneous magnetization) modify the range of energies and momenta that contribute to this diffusive enhancement of EEI, and thus modify QC.
Of the EEI corrections it is those of the Hartree-type via which the geometric gauge potential manifests itself. Here we shall focus on the EEI corrections to conductivity, for which the dominant contribution comes from processes characterized by the maximum number of diffusion poles. Following Ref. [10] we have calculated the Hartree correction to conductivity due to the interaction of electrons and holes with total spin 1 . This correction is determined by the contribution of the three-Diffuson processes. The two-Diffuson processes, each of them being the same order of magnitude as the three-Diffuson terms due to vector nature of the current vertices, compensate each other (see also Refs. 10,11).

In multiply-connected disordered samples the geometric vector-potential results in oscillations in the conductance with variation of the solid angle subtended by $\mathbf{M}$, (as well as in the density of states, and, in general, all quantities affected by the EEI correction in the Diffuson channel). At temperatures $T$ satisfying conditions $T \tau \gg 1$ and $L \geq L_{T}(\equiv \sqrt{D / T})$ the oscillating contribution to the conductance is given by

$$
\frac{e^{2} L_{T} \lambda_{1}}{2^{3 / 2} \pi \hbar L_{\perp}^{2}} \sum_{n=1}^{\infty} e^{-n \delta}(\sin n \delta-\cos n \delta) \cos n \Omega,
$$

where $\delta \equiv L / \sqrt{2} L_{T}$, and $\lambda_{1}$ (discussed in Ref. [10]) is a constant describing the interaction of an electron and hole with total spin 1. In ferromagnetic samples, Eq.(3) describes the contribution from $s$-electrons. As they are characterized by $M \tau \sim 0.1$, the triplet components of the Diffuson propagators are affected only moderately by the Zeeman (i.e. magnetization-induced) dynamical phase. For $d$-electrons such oscillations are very small in magnitude, because $M \tau \sim 15$ and hence the triplet Diffuson components are entirely suppressed. We emphasize that the oscillations described by Eq. (3) occur provided that not only is the condition for adiabaticity satisfied, but also the Zeeman dynamical phase does not suppress the correlation of electrons and holes (i.e. $M / T \leq 1$ ). We note that when the latter condition is fulfilled, the usual MR due to the suppression of the triplet-channel electron-hole interaction contribution 12,13 is absent.

Thus, for magnetizations having constant magnitude but varying direction, the geometric vector potential is seen to affect the conductivity in the regime where a homogeneous field of the same magnitude plays no role. This phenomenon arises not only in ferromagnets but also in generic conductors in the presence of arbitrary magnetic textures. In simply-connected settings geometricphase-induced anomalous magnetoresistance (due to spin effects) arises in regimes in which (spin) dynamical-phase contributions to magnetoresistance are negligible. In multiply-connected settings one has the striking situation in which inhomogeneous fields result in conductance oscillations. By contrast, weak homogeneous fields play no role, whereas stronger fields would lead only to anomalous magnetoresistance (and not oscillations). We note that orbital magnetic fields (which are rather strong in 
ferromagnets $\sim 0.1 T$ ) do not affect the dominant contribution to the EEI corrections, namely that arising from the Diffuson channel. As orbital magnetic fields suppress weak localization corrections, the oscillating EEI contribution can thus be distinguished from others. In addition, geometric phase effects can be disentangled.

Universal conductance fluctuations: We now consider stochastic variations in the conductance due to changes in the magnetization, i.e., universal conductance fluctuations (UCF) [15]. In order to address these UCF one can proceed by calculating the connected correlator $F\left(A_{\mathrm{g}}^{(1)}, \delta A_{\mathrm{g}}\right) \equiv\left\langle\sigma\left(A_{\mathrm{g}}^{(1)}\right) \sigma\left(A_{\mathrm{g}}^{(1)}+\delta A_{\mathrm{g}}\right)\right\rangle_{\mathrm{c}}$ between conductivities $\sigma$ at two different values of the geometric vectorpotential, say $A_{\mathrm{g}}^{(1)}$ and $A_{\mathrm{g}}^{(2)} \equiv A_{\mathrm{g}}^{(1)}+\delta A_{\mathrm{g}}$. Here, the angle brackets indicate an average over realizations of the disorder. We restrict our attention to the regime $L_{T} \ll L_{\phi}$. In this case, the contribution to the correlator arising from fluctuations in the diffusion coefficient [14] is essential, whereas the contribution arising from fluctuations in the density of states is negligible. Then, UCF in a ring with domain walls are described by the correlator

$$
\begin{aligned}
& F\left(A_{\mathrm{g}}^{(1)}, \delta A_{\mathrm{g}}\right)=\left(16 \pi^{2} / 3\right)\left(e^{2} / \hbar\right)^{2}\left(L_{T}^{2} / L^{3}\right) \\
& \quad \times \sum_{ \pm, i=\mathrm{C}, \mathrm{D}} L_{i}\left[1+2 \sum_{n=1}^{\infty} e^{-n L / L_{i}} \cos \left(\Omega \pm \frac{1}{2} \delta \Omega\right)\right],
\end{aligned}
$$

where $\delta \Omega$ is the difference between the solid angles corresponding to the two vector-potentials, $A_{\mathrm{g}}^{(1)}$ and $A_{\mathrm{g}}^{(2)}$, $\Omega$ is the average of these solid angles, and $L_{\mathrm{C}(\mathrm{D})}$ is the phase-breaking length for the Cooperon/Diffuson.

The implications of Eq. (4) for a single realization of the disorder are as follows. For simply-connected mesoscopic wires with no (spatially) transverse variations of $\mathbf{M} / M$, no electron trajectories enclose any geometric flux. Therefore variations in $\mathbf{M} / M$ lead to neither deterministic nor stochastic variations in the conductance. For simply-connected mesoscopic wires with transverse variations, flux can be enclosed and, therefore, there will be stochastic variations in the conductance. Indeed, there will effectively be a new value of the conductance after one quantum of geometric flux is added to the sample. For multiply-connected samples with no transverse variations, there will be conductance oscillations analogous to the $h / e$ (as well as $h / 2 e$ ) oscillations probed by Aharonov-Bohm quantal phases, familiar from mesoscopic physics. Finally, transverse variations in $\mathbf{M} / M$ will produce additional stochastic variations of the conductance, superposed on the $h / e$-like oscillatory variations. We note that for $d$-electrons the Diffuson contribution to UCF is suppressed because of the Zeeman interaction, whereas the Cooperon contribution persists. For $s$-electrons both the Cooperon and the Diffuson correlations are equally important for the UCF.

As for experimental realizations, an external magnetic field can be used to adjust the spatial pattern of $\mathbf{M} / M$, and thus alter the geometric flux. In this way, one should be able to probe variations in the conductance with the geometric flux. Topological excitations such as skyrmions also modulate quantum interference effects.

Acknowledgments: We thank B. L. Altshuler and $\mathrm{M}$. Weissmann for interesting and useful discussions, and N. Giordano for informing us of his research prior to publication. This work was supported by the Department of Energy, Division of Materials Sciences, under Award No. DEFG02-96ER45439 (YLG), and the National Science Foundation through grant DMR94-24511 (PMG).

[1] K. Hong and N. Giordano, Phys. Rev. B 51, 9855 (1995).

[2] K. Hong and N. Giordano, Resistance of a domain wall in a thin ferromagnetic wire, preprint (1997).

[3] At the level of classical theory the conductivity of ferromagnets having domain walls with a thickness much smaller than the electronic mean free path was addressed in the Boltzmann approximation by A. Cabrera and L. Falicov, Phys. Stat. Solidi (b) 61, 539 (1974).

[4] The idea of seeking implications of geometric (and related) phases in the context of mesoscopic phenomena has been considered quite widely [6, []. The present setting, viz., that of ferromagnetic wires with domain walls, may provide a viable route for realising such effects.

[5] M. Berry, Proc. R. Soc. London A 392, 45 (1984).

[6] D. Loss, P. M. Goldbart and A. V. Balatsky, Phys. Rev. Lett. 65, 1655 (1990); A. Stern, Phys. Rev. Lett. 68, 1022 (1992) H. Mathur and A. D. Stone Phys. Rev. Lett. 68, 1964 (1992); A. G. Aronov and Y. Lyanda-Geller, Phys. Rev. Lett. 70, 343 (1993).

[7] D. Loss, H. Schoeller and P. M. Goldbart, Phys. Rev. B 48, 15218 (1993).

[8] G. Tatara, H. Fukuyama, Phys. Rev. Lett. 78, 3773 (1997).

[9] In the Cooperon propagator the net spin is defined to be the total spin of the particles whose correlation is being described; in the Diffuson propagator the net spin is defined to be the difference of these spins.

[10] B. L. Altshuler and A. G. Aronov, in Electron-Electron Interactions in Disordered Systems, A. L. Efros and M. Pollak (Eds.), p. 11, (North-Holland, Amsterdam, 1985).

[11] H. Fukuyama, ibid., p. 155.

[12] P. Lee, T. Ramakrishnan, Phys. Rev. B 26, 4009 (1982).

[13] B. L. Altshuler, A. G. Aronov and A. Yu. Zyuzin, Solid State Comm. 44, 137 (1982).

[14] B. L. Altshuler and B. I. Shklovskii, Sov. Phys. JETP 64, 127 (1986).

[15] In Ref. 8 UCF were addressed by drawing an analogy with mesoscopic fluctuations due to motion of a single atom [16]. The present work shows that whether or not changes in the magnetization lead to changes in the conductance depends rather sensitively on the geometrical properties of the magnetization configuration in question.

[16] B. L. Altshuler and B. Z. Spivak, JETP Lett. 42, 447 (1985); S. Feng, P. Lee and A. D. Stone, Phys. Rev. Lett. 56, 1960 (1986). 